\title{
Development and validation of the Kidney Transplantation and Quality of Life, a Korean questionnaire to assess the general quality of life and other health issues associated with medication change in kidney transplant recipients
}

\author{
Jinsoo Rhu', Kyo Won Lee', Young Jae Chung', Jae Berm Park', Ju Young Choi', \\ Sung Joo Kim ${ }^{1}$, Jin Sang Jung ${ }^{2}$, Sehyun $\mathrm{Kim}^{3}$ \\ ${ }^{1}$ Department of Surgery, Samsung Medical Center, Sungkyunkwan University School of Medicine, Seoul, Korea; \\ ${ }^{2}$ Astellas Pharma Korea Inc., Seoul, Korea; \\ ${ }^{3}$ Graduate School, Dankook University, Yongin, Korea
}

\begin{abstract}
Background: This study aimed to develop and validate the Kidney Transplantation and Quality of Life (KTQoL) questionnaire to evaluate the quality of life (QoL) in Korean kidney transplantation (KT) patients. During the validation, the KTQoL was used to compare the QoL of KT patients before and after conversion from twice-daily to a once-daily regimen of tacrolimus. Methods: Construct and content validity of the 24-item KTQoL was evaluated using factor analysis and a panel of experts, respectively. The questionnaire was validated in $50 \mathrm{KT}$ patients, conducted before and after conversion from twice-daily to once-daily tacrolimus. Internal consistency was evaluated based on Cronbach's alpha coefficient.

Results: The KTQoL showed high internal consistency (Cronbach's alpha 0.71 to 0.88 ). Besides the Concerns category, both the general and specific QoL of KT patients were relatively good ( $270 \%$ of positive responses) and did not change markedly after conversion to the once-daily regimen $(42.9 \pm 8.8$ vs. $43.6 \pm 8.6, \mathrm{P}=0.740)$. After conversion, men scored better than women in total KTQoL, Specific QoL, and Symptoms, while employed patients had better Daily Life scores and showed greater improvement in Daily Life scores compared with unemployed patients.

Conclusions: The KTQoL seems to be a reliable instrument to evaluate general and specific QoL in Korean KT patients. Most patients evaluated their QoL positively. Conversion to once-daily tacrolimus had no significant effect on QoL in the total sample of KT patients. The QoL of men and/or employed persons might improve more after conversion to once-daily tacrolimus.
\end{abstract}

Keywords: Medication adherence; Kidney transplantation; Quality of life; Validation studies; Surveys; Surveys and questionnaires; Psychometrics

\section{INTRODUCTION}

Kidney transplantation (KT) is the most efficient treatment option for patients with end-stage renal disease

Received October 23, 2019

Revised December 25, 2019

Accepted December 27, 2019

Correspondence to: Sehyun Kim

Graduate School, Dankook University, 152 Jukjeon-ro, Suji-gu, Yongin 16890, Korea

Tel: +82-31-8005-3952, Fax: +82-2-3448-0511

E-mail: kim.ssam@gmail.com concerning both survival and quality of life (QoL). Immunosuppression is essential for kidney graft survival, and maintenance immunosuppressive therapies have contributed to a substantial decrease in early graft failure rates [1]. However, long-term graft survival is still a concern because of the relatively common problem of immune-mediated allograft rejection [2]. Available evidence suggests that the allograft rejection may be, at least partially, associated with poor compliance of KT patients with immunosuppressive therapies [3]. Therefore,

This is an Open Access article distributed under the terms of the Creative Commons Attribution Non-Commercial License (http://creativecommons. org/ licenses/by-nc/4.0/) which permits unrestricted non-commercial use, distribution, and reproduction in any medium, provided the original work is properly cited. 


\section{HIGHLIGHTS}

- Kidney Transplantation and Quality of Life (KTQoL) seems to be a reliable instrument to evaluate general and specific quality of life (QoL) in Korean kidney transplantation (KT) patients.

- Most KT patients evaluated their QoL positively except for the concerns subdomain.

- Conversion from the twice-daily to the once-daily tacrolimus regimen had no significant effect on QoL determined with the KTQoL in the total group of KT patients.

new formulations of immunosuppressants, suitable for once-daily administration, have been developed to overcome this issue. The use of these novel regimens, such as once-daily tacrolimus, is associated with better medication compliance and fewer adverse effects in KT patients [4].

In modern medicine, considering treatment outcomes solely in terms of objective improvement in medical status is no longer sufficient. Physicians must also consider subjective improvement in QoL, defined as the perceived influence of a disease and its treatment on the patient's physical, mental and emotional status, socioeconomic situation, spiritual and functional well-being, satisfaction with therapy, and the domains of sexual experiences, intimacy, and body self-image [5-7]. Importantly, higher QoL is associated with greater adherence [8-11].

Many instruments are available to measure QoL; however, the vast majority are generic scales that do not necessarily measure the burden associated with a specific condition accurately. A few QoL scales developed specifically for KT patients or individuals with a kidney disease are also available, such as the Kidney Transplant Questionnaire (KTQ) [12], the Kidney Disease Questionnaire (KDQ) [13], the Kidney Disease Quality of Life Instrument (KDQoL) [14], and the End-Stage Renal Disease Symptom Checklist-Transplantation Module (ESRDSCTM) [15]. However, these instruments were mostly developed in Western countries, which limits their applicability to Korean KT patients owing to the well-known problems with the cross-cultural adaptation of such scales [16-18].

This study aimed to develop and validate the Kidney Transplantation and Quality of Life (KTQoL) questionnaire, specifically intended to evaluate Korean KT patients' QoL. As a part of the validation process, the KTQoL was used to compare the QoL of KT patients before and after conversion from twice-daily to a oncedaily regimen of tacrolimus.

\section{METHODS}

\section{Ethical Standards}

The study was conducted as per Good Clinical Practice and the ethical principles of the Helsinki Declaration. The Institutional Review Board of the Samsung Medical Center approved the study protocol (IRB No. SMC 2014-11-082). Written consent was obtained from each participant before data collection or interview. The study was registered at clinicaltrials.gov (NCT03623217).

\section{Research Methods}

A combined method approach was used to develop and validate the questionnaire. Qualitative methods and quantitative analysis methods were used during the questionnaire development phase and questionnaire validation process, respectively. Patients were eligible to participate if they had received twice-daily tacrolimus (Prograf; Astellas Pharma Inc., Toyama, Japan) for at least 6 months and up to 12 months after kidney transplantation and then converted to the once-daily formulation (Advagraf; Astellas Pharma Inc.).

\section{Questionnaire development}

The questionnaire development phase included six patients according to the following criteria: one patient aged 30-39 years, one patient aged 60-69 years, one employed patient, one unemployed patient, one patient receiving multiple concomitant medications, and one patient receiving few concomitant medications (based on the investigator's judgment). A theoretical thematic analysis [19] was conducted based on the research question "How should health-related quality of life in kidney transplant recipients be measured?" as follows: (1) questionnaire 
domains were created to analyze themes; (2) in-depth interviews were conducted with the six participants and transcribed verbatim; (3) questionnaire items were generated for each domain; (4) qualitative item reduction processes were carried out; (5) the identified items were revised to create a self-administered questionnaire, with each item scored using a three-point or five-point Likert scale; (6) a cognitive debriefing interview and linguistic validation were conducted on a preliminary questionnaire with a focus group of participants, followed by further item reduction and modifications; and (7) a pilot questionnaire was prepared for field testing.

\section{Questionnaire validation}

In total, 50 patients meeting the following criteria were recruited as participants for the pilot field test: (1) aged 19 to 65 years, (2) currently taking twice-daily tacrolimus for at least 6 months, and (3) scheduled for conversion to once-daily tacrolimus. The draft questionnaire was administered to the participants twice: the first time during treatment with twice-daily tacrolimus and the second time during the first or second month of treatment with once-daily tacrolimus (between 20 and 51 days after conversion). Questions in the medication change category were included only in the second survey. The questionnaire's construct validity was evaluated through factor analysis, while its content validity was evaluated by a panel of experts comprising investigators in the medical or scientific field of organ transplantation. The internal consistency of the questionnaire items was evaluated based on Cronbach's alpha coefficient [20,21].

\section{Statistical Analysis}

All statistical analyses were performed using IBM SPSS ver. 24.0 (IBM SPSS Corp., Armonk, NY, USA), with statistical significance defined as $\mathrm{P}<0.05$. No formal sample size calculations were done.

\section{Validation of the questionnaire}

Scores of individual questionnaire items were factoranalyzed, and promax oblique rotation was applied. Items with low loading value $(<0.4)$ were treated as irrelevant. Internal consistency of the questionnaire items was evaluated using Cronbach's alpha coefficient; if the coefficient was 0.7 or higher, internal consistency was considered as achieved. A team of nine investigators evaluated the individual item validity from a content perspective using a four-point Likert scale (1, irrelevant; 2 , relevant with significant revision; 3 , relevant with minor revision; 4 , very relevant). Items scored as 3 or 4 in $75 \%$ or more instances, were regarded as relevant. Irrelevant items were removed.

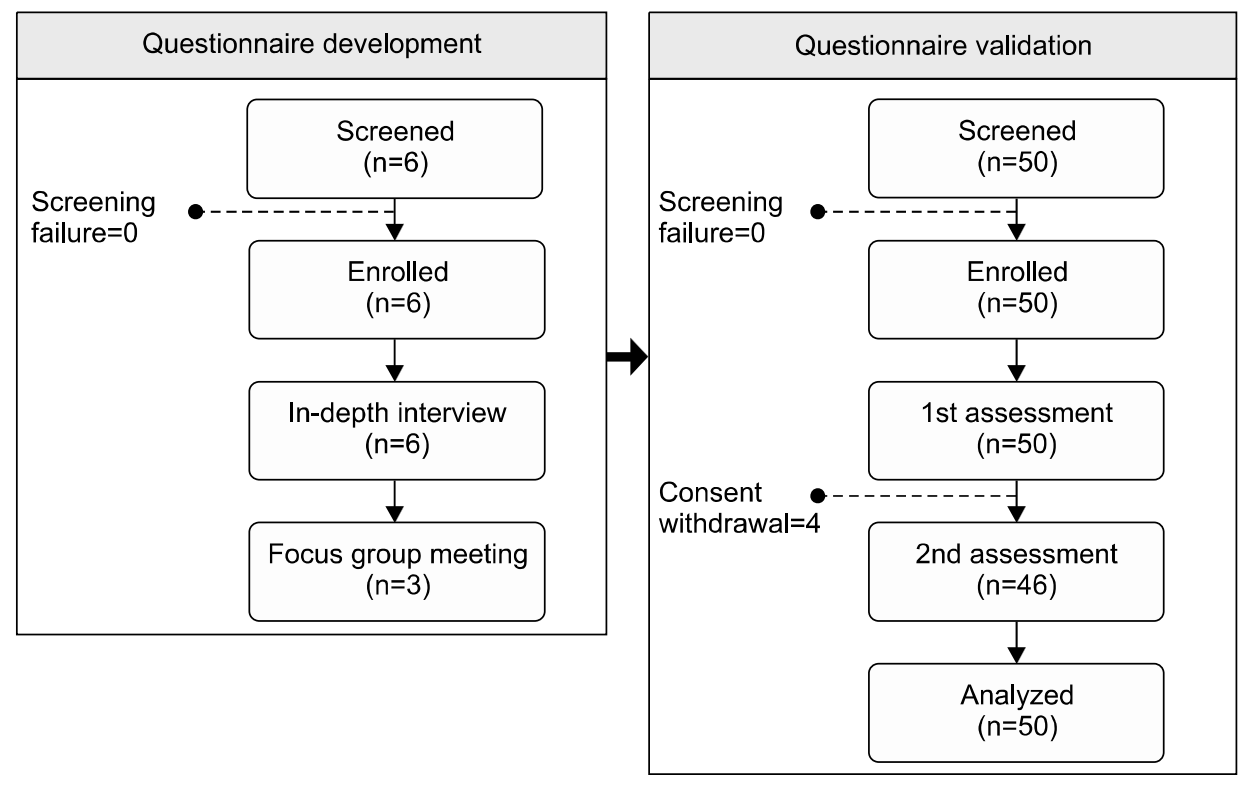

Fig. 1. Subject distribution. In total, six patients participated in the in-depth interview, of whom three were randomly chosen to be invited to the focus group meeting. For the quantitative research phase, 50 patients were screened and enrolled. All except four patients completed two site visits to answer the questionnaire. The analysis included all available data. 


\section{Evaluation of QoL after medication change}

Descriptive statistics were calculated for the scores obtained from the two administrations of the questionnaire in the pilot field test. A successive positive integer was assigned to each category on the scale (starting from 0 ) to facilitate data analysis. Reverse scoring was applied for certain items to assign a higher score for more positive answers. Paired t-tests were carried out on the summed scores for each domain. As an exploratory analysis, "proportion of positive responses," defined as the proportion of participants responding with scores of 1 or 2 on the three-point Likert scale or scores of 4 or 5 on the five-point Likert scale, was calculated for individual items. No imputations were done for missing data.

\section{RESULTS}

\section{Participants}

Each step of the study was sequentially conducted from February 2015 to October 2017 (Fig. 1). As planned for the questionnaire development phase, six participants satisfying the pre-determined criteria were enrolled. Of these, three participants were randomly chosen for the focus group meeting. For the pilot field test, 50 participants completed the questionnaire during treatment with

Table 1. Demographic data of participants in the pilot field study

\begin{tabular}{lc}
\hline \multicolumn{1}{c}{ Variable } & Value \\
\hline Age $(\mathrm{yr})$ & \\
Mean \pm SD & $50.2 \pm 11.1$ \\
Median (range) & $52(24-67)$ \\
Sex, n (\%) & $21(42)$ \\
Male & $29(58)$ \\
Female & $1,525 \pm 549.06$ \\
Days since KT & $1,504(524-3,240)$ \\
Mean \pm SD & \\
Median (range) & $22(44)$ \\
Employment status, n $(\%)$ & $28(56)$ \\
Unemployed & \\
Employed &
\end{tabular}

A total number of 50 participants were analyzed. Mean \pm SD ages of men and women were $53.7 \pm 10.3$ years and $47.7 \pm 11.3$ years, respectively. The proportions of employed men and women were $67 \%(14 / 21)$ and $48 \%(14 / 29)$ respectively.

$\mathrm{SD}$, standard deviation; KT, kidney transplantation. twice-daily tacrolimus, and 46 participants completed it during treatment with once-daily tacrolimus (Fig. 1). Mean \pm standard deviation (SD) age of participants was $50.2 \pm 11.1$ years; time since KT ranged from 524 to 3,240 days. Women and employed persons accounted for $58 \%$ and $56 \%$ of the sample, respectively (Table 1 ).

\section{Questionnaire Development and Validation Pilot development}

Based on the initial conceptual framework, five categories were created: Q1, Overall Health before medication change; Q2, Concerns and Symptoms; Q3, Daily Life; Q4, Overall Health after medication change; Q5, issues associated with medication change. In total, 37 items were drafted after an in-depth review with six participants. After eliminating two items through cognitive debriefing and linguistic validation conducted with the focus group, a pilot version of the questionnaire comprising 35 items was prepared.

\section{Construct and content validity}

Item response rate (mean $\pm \mathrm{SD}$ ) was $99.69 \% \pm 0.74 \%$ and $99.63 \% \pm 0.98 \%$ at the first and second assessments of the field test, respectively. First-order factor analysis was conducted based on the following three areas: ques-

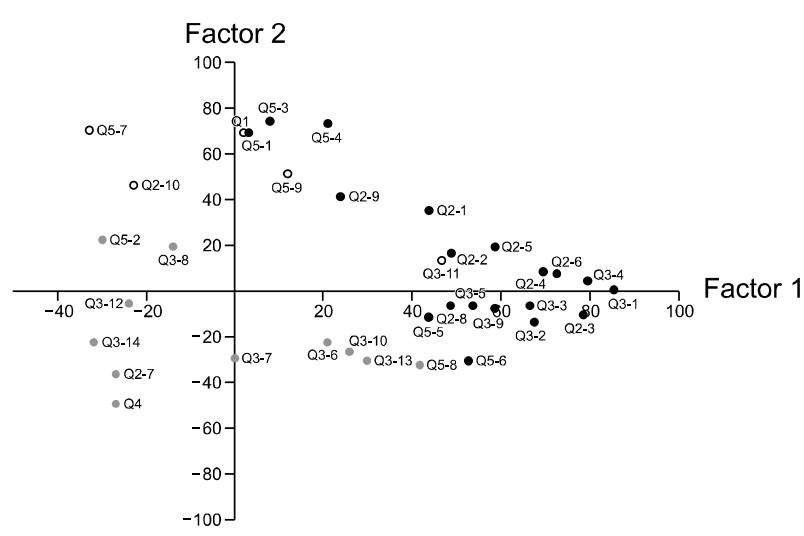

Fig. 2. Scatter plot of loading values from factor analysis. Each item was plotted based on the factor loading value $\times 100$ for each factor. If the estimated loadings were $>0.4$ or $<-0.4$, the items associated with these loadings were identified as significant (blue or orange dots). Items plotted with gray dots were removed from the questionnaire. Items plotted with orange dots were treated with the reverse scoring method (plotted by $-1 \times$ the original loading value $\times 100$ ). 
tions on anxiety or worry, discomfort, or pain after surgery; questions on daily life; questions on changing immunosuppressive regimen. As a result, two factors were identified as meaningful. Linear transformation of factors was applied to improve interpretability, and factor loadings were obtained after promax rotation (Fig. 2). Eleven items were discarded based on factor analysis and qualitative judgment by the investigators (refer to gray circles in Fig. 2). The remaining 24 items received $100 \%$ positive evaluation ( $3=$ relevant with minor revision or $4=$ very relevant) by the expert panel. Based on the factor analysis, seventeen items were classified under General QoL domain measuring general QoL of patients following kidney transplant surgery, while seven items were classified under Specific QoL domain measuring the QoL of patients after conversion from twice-daily tacrolimus to once-daily tacrolimus.

\section{Internal consistency}

Cronbach's alpha coefficients for the 17 items of the General QoL domain were 0.87 and 0.88 when tacrolimus was taken twice-daily and once-daily, respectively, indicating internal consistency (Table 2). Cronbach's alpha coefficient for the 7 items of the Specific QoL domain associated with medication change from twice-daily to once-daily was 0.71 , again indicating internal consistency (Table 2). Based on the clinical judgment, the General QoL items were classified into three subdomains (Concerns, Symptoms, and Daily Life) and Cronbach's alpha coefficient was computed for each subdomain. The Concerns and Symptoms subdomain items showed acceptable internal consistency; however, the Cronbach's alpha coefficient for the Daily Life subdomain items was $<0.7$ for both twice-daily and once-daily administration of tacrolimus (Table 2). Finally, these 24 items were included in the self-administered questionnaire, newly entitled as the KTQoL questionnaire (Table 3). The subscore for General QoL domain ranges from 0 to 58 and the subscore for the Specific QoL related to medication change ranges from 0 to 22 ; higher scores indicate better QoL.

\section{QoL in Korean Kidney Transplant Recipients}

Based on the participants' responses to the final 17 items in the General QoL domain, no significant change in the mean score was observed between the twice-daily and once-daily measurement time points (42.9 vs. 43.6, $\mathrm{P}=0.740$ ) (Fig. 3A). A subgroup analysis based on employment status or sex was also performed. In the first assessment done during treatment with twice-daily tacrolimus, no significant difference in the General QoL domain score was detected between men and women (Fig. 3B). However, during treatment with once-daily tacrolimus, men had a higher mean score in the General QoL domain than women (46.8 vs. 41.2, $\mathrm{P}=0.019$ ) (Fig. 3B). Additionally, men had a higher mean score in the Symptoms subdomain during treatment with once-daily tacrolimus than women ( 24.8 vs. $22.3, \mathrm{P}=0.012)$. No significant differences were observed in the General QoL domain between employed and unemployed participants with either twice- daily or once-daily tacrolimus (Fig. $3 \mathrm{C}$ ). However, the mean score for the Daily Life subdomain was higher among employed than unemployed participants (7.3 vs. $6.2, \mathrm{P}=0.037$ ), as was the change

Table 2. Cronbach's alpha of KTQoL factors

\begin{tabular}{llccc}
\hline \multicolumn{1}{c}{ Factor } & \multicolumn{1}{c}{ Item } & No. of items & $\begin{array}{c}\text { First assessment } \\
\text { (twice-daily) }\end{array}$ & $\begin{array}{c}\text { Second assessment } \\
\text { (once-daily) }\end{array}$ \\
\hline $\begin{array}{l}\text { General QoL } \\
\text { General QoL subdomain }\end{array}$ & Q1, Q2 subset, Q3 subset & 17 & 0.87 & 0.88 \\
Concern & Q2-3, Q2-4, Q2-5, Q2-6 & & & 0.93 \\
Symptoms & Q2-1, Q2-2, Q2-10, Q3-2, Q3-3, Q3-4, Q3-5, Q3-9 & 8 & 0.75 & 0.92 \\
$\begin{array}{l}\text { Daily life } \\
\text { Specific QoL }\end{array}$ & Q2-8, Q2-9, Q3-1, Q3-11 & 4 & 0.39 & 0.63 \\
\hline
\end{tabular}

KTQoL, Kidney Transplantation and Quality of Life; QoL, quality of life. 
Table 3. Complete list of the KTQoL items

\begin{tabular}{|c|c|c|}
\hline Item & Question & Response scale \\
\hline $\mathrm{Q1}^{\text {a) }}$ & How do you evaluate your current health overall? & $\begin{array}{l}\text { (1) Very good (2) Good (3) Moderate (4) Poor } \\
\text { (5) Very poor }\end{array}$ \\
\hline Q2 & $\begin{array}{l}\text { Frequency of the following feelings/conditions during the past } 1 \text { month: } \\
\text { anxiety, concerns, discomfort, or pain associated with transplant surgery }\end{array}$ & \\
\hline Q2-1 & $\begin{array}{l}\text { How often have you experienced pain or discomfort over the transplant } \\
\text { surgical site? }\end{array}$ & $\begin{array}{l}\text { (1) Always (2) Mostly (3) Sometimes (4) } \\
\text { Rarely (5) Never }\end{array}$ \\
\hline Q2-2 & How often have you experienced nervousness after the transplant surgery? & $\begin{array}{l}\text { (1) Always (2) Mostly (3) Sometimes (4) } \\
\text { Rarely (5) Never }\end{array}$ \\
\hline Q2-3 & $\begin{array}{l}\text { How often have you worried about potential infection due to } \\
\text { administration of immunosuppressants after the transplant surgery? }\end{array}$ & $\begin{array}{l}\text { (1) Always (2) Mostly (3) Sometimes (4) } \\
\text { Rarely (5) Never }\end{array}$ \\
\hline Q2-4 & $\begin{array}{l}\text { How often have you worried about how well the kidney graft function } \\
\text { will be maintained? }\end{array}$ & $\begin{array}{l}\text { (1) Always (2) Mostly (3) Sometimes (4) } \\
\text { Rarely (5) Never }\end{array}$ \\
\hline Q2-5 & $\begin{array}{l}\text { How often do you worry about having bad results from laboratory tests } \\
\text { done during clinic visits? }\end{array}$ & $\begin{array}{l}\text { (1) Always (2) Mostly (3) Sometimes (4) } \\
\text { Rarely (5) Never }\end{array}$ \\
\hline Q2-6 & $\begin{array}{l}\text { How often do you worry about how long your health will be maintained } \\
\text { after the transplant surgery? }\end{array}$ & $\begin{array}{l}\text { (1) Always (2) Mostly (3) Sometimes (4) } \\
\text { Rarely (5) Never }\end{array}$ \\
\hline Q2-8 & $\begin{array}{l}\text { How much of a financial burden do you have in association with } \\
\text { prescribed medication after the surgery? }\end{array}$ & (1) Very much (2) Some (3) Not at all \\
\hline Q2-9 & How much discomfort do you experience in visiting the clinic regularly? & (1) Very much (2) Some (3) Not at all \\
\hline Q2-10 & $\begin{array}{l}\text { How much more comfortable do you feel with breathing after the } \\
\text { transplant surgery than before? }\end{array}$ & (1) Very much (2) Some (3) Not at all \\
\hline Q3 & Questions related to daily life; responses should refer to frequencies. & \\
\hline Q3-1 & $\begin{array}{l}\text { How often do you feel discomfort in your daily life after the transplant } \\
\text { surgery? }\end{array}$ & $\begin{array}{l}\text { (1) Always (2) Mostly (3) Sometimes (4) } \\
\text { Rarely (5) Never }\end{array}$ \\
\hline Q3-2 & $\begin{array}{l}\text { How often do you experience depression or dispirited after the transplant } \\
\text { surgery? }\end{array}$ & $\begin{array}{l}\text { (1) Always (2) Mostly (3) Sometimes (4) } \\
\text { Rarely (5) Never }\end{array}$ \\
\hline Q3-3 & How often do you feel dizziness after the transplant surgery? & $\begin{array}{l}\text { (1) Always (2) Mostly (3) Sometimes (4) } \\
\text { Rarely (5) Never }\end{array}$ \\
\hline Q3-4 & How often do you have problems sleeping after the transplant surgery? & $\begin{array}{l}\text { (1) Always (2) Mostly (3) Sometimes (4) } \\
\text { Rarely (5) Never }\end{array}$ \\
\hline Q3-5 & $\begin{array}{l}\text { How often do you have post-meal stomach discomfort after the transplant } \\
\text { surgery? }\end{array}$ & $\begin{array}{l}\text { (1) Always (2) Mostly (3) Sometimes (4) } \\
\text { Rarely (5) Never }\end{array}$ \\
\hline Q3-9 & Do you have any discomfort with eating? & (1) Very much (2) Some (3) Not at all \\
\hline Q3-11 a) & How much has your daily life improved after the transplant surgery? & (1) Very much (2) Some (3) Not at all \\
\hline Q5 & $\begin{array}{l}\text { Questions related to change of immunosuppressants; responses should } \\
\text { refer to frequencies. }\end{array}$ & \\
\hline Q5-1 & $\begin{array}{l}\text { How much pain or physical discomfort have you experienced since } \\
\text { change of the medication? }\end{array}$ & $\begin{array}{l}\text { (1) Always (2) Mostly (3) Sometimes (4) } \\
\text { Rarely (5) Never }\end{array}$ \\
\hline Q5-3 & $\begin{array}{l}\text { Since changing the medication, how often do you worry about the } \\
\text { efficacy of the new medication? }\end{array}$ & $\begin{array}{l}\text { (1) Always (2) Mostly (3) Sometimes (4) } \\
\text { Rarely (5) Never }\end{array}$ \\
\hline Q5-4 & $\begin{array}{l}\text { How often do you worry about whether there will be any problem } \\
\text { with your kidney graft due to the new medication? }\end{array}$ & $\begin{array}{l}\text { (1) Always (2) Mostly (3) Sometimes (4) } \\
\text { Rarely (5) Never }\end{array}$ \\
\hline Q5-5 & How often do you forget to take the immunosuppressants? & $\begin{array}{l}\text { (1) Always (2) Mostly (3) Sometimes (4) } \\
\text { Rarely (5) Never }\end{array}$ \\
\hline Q5-6 & $\begin{array}{l}\text { Besides the medicine prescribed by the hospital, are there any medicines } \\
\text { that you take for your health? }\end{array}$ & (1) A lot (2) Some (3) Not at all \\
\hline Q5-7 & How much expectation do you have about the new medication? & (1) Very much (2) Some (3) Not at all \\
\hline Q5-9 & How much do you feel the new medication is convenient to take? & (1) Very much (2) Some (3) Not at all \\
\hline
\end{tabular}

KTQoL, Kidney Transplantation and Quality of Life.

${ }^{a)}$ Reverse scoring should be applied. 
General QoL
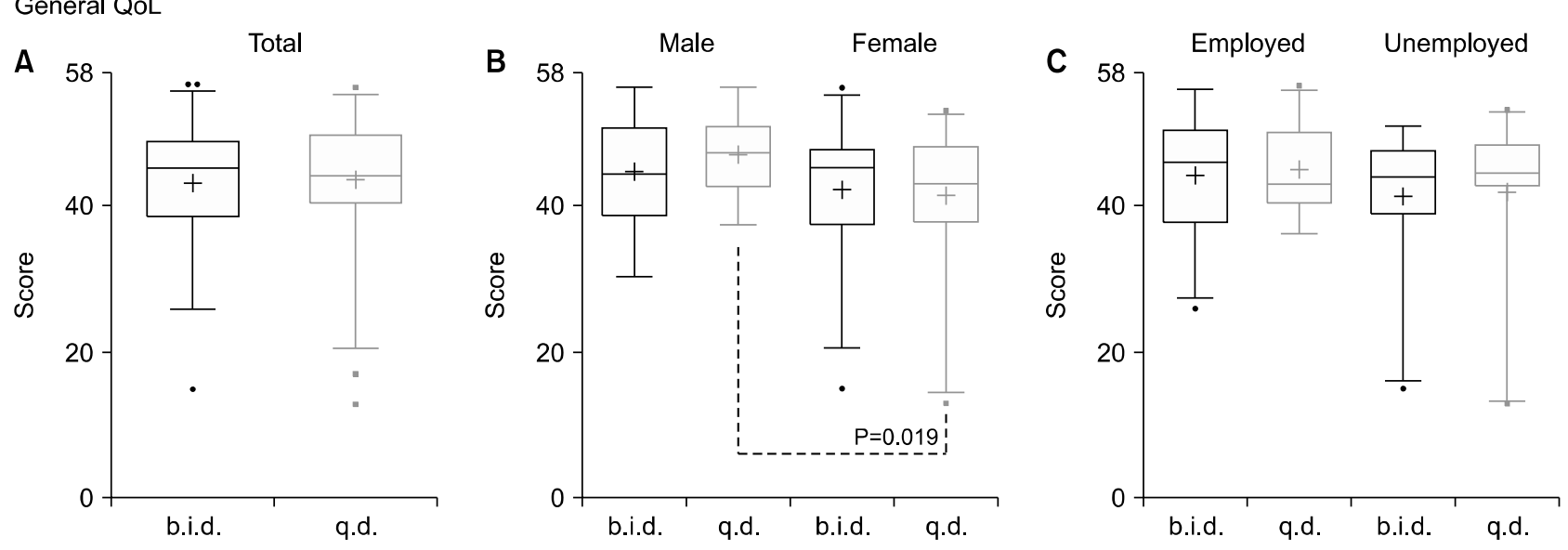

Specific QoL
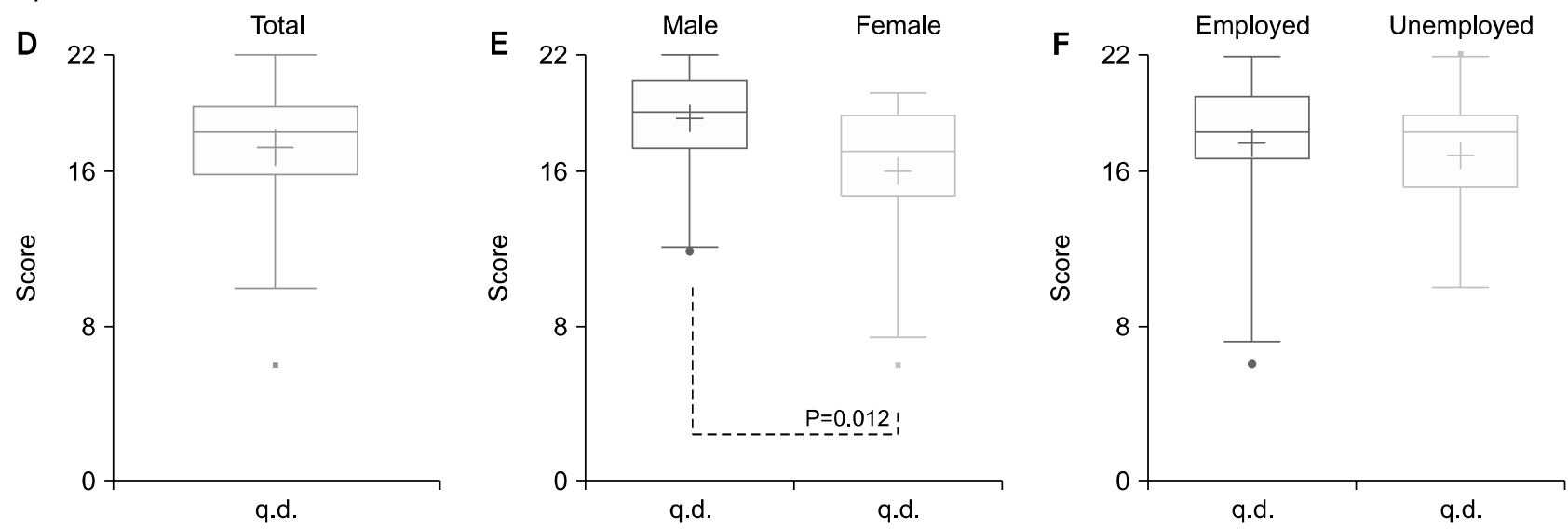

Fig. 3. Distribution of quality of life (QoL) scores. The middle line in the box indicates median, + sign indicates mean, and whiskers indicate 5th and 95th percentiles. b.i.d., twice daily; q.d., once daily.

in the mean score for Daily Life (0.60 vs. -0.55 , $\mathrm{P}=0.005)$.

Regarding Specific QoL associated with the change from twice-daily to once-daily tacrolimus, the mean \pm SD score was $17.2 \pm 3.6$, men had higher scores than women (18.7 vs. 16.0, $\mathrm{P}=0.012$ ), and no significant difference was observed between employed and unemployed participants (Fig. 3D-F).

Based on the proportion of positive responses, approximately $70 \%$ of the participants assessed their overall health as "good" or "very good"; $72 \%$ and $67 \%$ during the twice-daily and once-daily regimen, respectively. The proportion of positive responses to the Symptoms subdomain items was relatively high, ranging from $80 \%$ to $94 \%$ during the twice-daily regimen and $80 \%$ to $98 \%$ during the once-daily regimen. The proportion of pos- itive responses to the Concerns subdomain items ranged from $56 \%$ to $65 \%$ during the twice-daily regimen and $59 \%$ to $72 \%$ during the once-daily regimen. Among the four items in the Daily life subdomain, "convenience in daily life" received a high percentage of positive responses; $98 \%$ and 96\% during the twice-daily and once-daily regimen, respectively. The proportion of positive responses to the other three items in the Daily life subdomain ranged from $72 \%$ to $80 \%$ during the twice-daily regimen and $72 \%$ to $89 \%$ during the once-daily regimen. Compared with the General QoL results, items assessing concerns or symptoms after medication change in the Specific QoL domain received a similar proportion of positive responses: $73 \%$ to concerns about drug efficacy, $65 \%$ to concerns about kidney function, and $87 \%$ to pain/uneasy feelings. Compliance with 
immunosuppressants was good; $85 \%$ and $15 \%$ of participants never or rarely skipped immunosuppressants, respectively. Only $2 \%$ of participants reported using other medications; $91 \%$ of participants gave positive responses for convenience and expectations on once-daily tacrolimus.

\section{DISCUSSION}

This study demonstrated good psychometric characteristics for the KTQoL questionnaire, an original Korean questionnaire evaluating general QoL and specific QoL related to conversion from twice-daily to once-daily tacrolimus in KT patients. The 24-item questionnaire demonstrated a high internal consistency rate (Cronbach's alpha, 0.71 to 0.88 ), and its validity was confirmed by both factor analysis and expert judgment, thereby confirming the appropriateness of the multi-step development process. Although the Cronbach's alpha for the Daily Life subdomain was relatively low (0.39 and 0.63 from the first and second assessments, respectively), no items were removed from this domain since the value was still above the accepted threshold level (0.3) warranting further analysis [22]. Since the daily life of patients can be affected by many factors that were not considered in the present study, the validity of each subdomain should be further verified in future research. One important advantage of the KTQoL questionnaire is the short time (approximately 10 minutes) required to complete it, which cannot be undervalued in a busy clinical setting. The only currently available adapted instrument validated for the evaluation of QoL in Korean patients with kidney disease, the KDQoL-SF, comprises about 80 items and requires about 16 minutes to complete [23]. Additionally, the KTQoL, although brief, addresses some vital issues related to general and specific $\mathrm{QoL}$ of $\mathrm{KT}$ patients, including overall health, concerns, symptoms, and daily life.

Although several general and specific questionnaires evaluating QoL of KT patients are available, including the $\mathrm{KTQ}$, KDQ, KDQoL-SF, ESRDSC-TM, and generic 36-Item Short Form Survey [12-15, 24], all these instruments were developed in Western countries. For use in the Korean population, they not only need to be translated but also subjected to a time-consuming and not always effective process of cross-cultural adaptation [16-18]. Previous experience with self-reported instruments analyzing the QoL of KT patients and persons with other medical conditions suggests that the process of cross-cultural adaptation may be challenging [6,25,26]. For example, a clear-cut distinction between treatment in an inpatient and outpatient setting exists in the Western healthcare systems, whereas, in Korea all healthcare institutions are referred to in lay language as "hospitals," regardless of whether they provide inpatient care or consultation only. A good example of cultural, linguistic, and cognitive differences between Englishand Korean- speaking people is the Q5-6 of the KTQoL "Besides the medicine prescribed by the hospital, are there any medicines that you take for your health? " referring to medications prescribed at the hospital rather than to the medications prescribed by a physician, as used commonly in Western questionnaires.

Considering all the above, we decided to develop a dedicated instrument to evaluate QoL in Korean KT patients, instead of adapting a questionnaire available in English. Although the KTQoL questionnaire is unsuitable for international multicenter studies and comparative analyses, it is tailored for the needs of the Korean KT patients, which is of utmost importance from the clinicians' perspective in Korea.

Aside from the psychometric validation, another step in the development process of the KTQoL, crucial in the context of its clinical application, was the analysis of changes in QoL associated with converting KT patients from twice-daily to a once-daily regimen of tacrolimus. A major risk of long-term graft failure is the de novo formation of donor-specific human leukocyte antigen antibodies (dnDSA) [2]. Since nonadherent patients are more likely than adherent patients to develop dnDSA [3] and the use of once-daily tacrolimus is associated with better adherence [4], the conversion to this regimen is expected to be crucial in preventing de novo DSA formation and graft failure.

Using the new questionnaire, the KTQoL, the present study demonstrated that both the General and Specific QoL of Korean KT patients were relatively good and did 
not change considerably after conversion to the once-daily regimen. The proportion of positive responses to the Symptoms subdomain items was relatively high, but lower for the Concerns subdomain. A subgroup analysis, with the QoL scores stratified according to participants' sex and employment status, provided some clinically relevant data. The analysis demonstrated that after conversion to the once-daily regimen, men and employed patients had significantly higher QoL scores in some domains compared with women and unemployed patients. Specifically, men scored higher than women on overall General QoL, Specific QoL, and the Symptoms subdomain of General QoL, whereas employed participants scored higher on the Daily Life subdomain and showed a greater increase in Daily Life scores than did unemployed patients. Although men had a higher employment rate in this study than women (67\% vs. $48 \%$ ), the number of subjects in each subgroup was too small to speculate about the exact role of sex and employment as determinants of QoL. When interpreting these findings, it should be considered that some studies, albeit not all, have demonstrated that male patients and employed patients are generally more likely to be less adherent than women or unemployed patients (for review, see [27]). However, due to the correlation between employment and sex (i.e., higher employment in men), these results require further study to analyze the benefit of conversion from once-daily to twice-daily on specific patient groups. Nevertheless, the observation that conversion from a twice-daily to a once-daily regimen contributed to higher QoL scores in some domains might be clinically meaningful and therefore, should be verified by a large-scale study using the KTQoL and a validated compliance tool (e.g., the Morisky Medication Adherence Scale, MMAS-8) [28].

This study had some potential limitations. Since our research primarily aimed to validate the newly developed instrument, the study sample was too small and statistical analyses were too limited to conclude possible benefits of conversion from twice-daily to once-daily tacrolimus on the Korean KT patients' QoL. Further, the results suggest that the improvement in QoL might be observed solely in some specific groups of KT patients, and hence, future studies should verify whether QoL of this population is modulated by other established sociodemographic determinants besides sex and employment status, such as educational level and social support [29]. Future research should also verify whether the use of the KTQoL can be expanded to other cohorts, e.g., patients on dialysis. Finally, it needs to be emphasized that content validity of the KTQoL was evaluated solely by healthcare providers and not by patients.

In conclusion, this study provided some meaningful observations summarized as follows: (1) the KTQoL seems to be a reliable instrument to evaluate the general and specific QoL of Korean KT patients; (2) except for the Concerns subdomain, most KT patients evaluated their QoL positively; and (3) conversion from the twice-daily to the once-daily tacrolimus regimen had no significant effect on the QoL of the whole sample as determined using the KTQOL.

\section{ACKNOWLEDGMENTS}

\section{Conflict of Interest}

This study was funded by Astellas Pharma Korea, Inc. All authors report nonfinancial support from Astellas Pharma Korea, Inc. JSJ is employee of Astellas Pharma Korea, Inc. No other potential conflict of interest relevant to this article was reported.

\section{Funding/Support}

This study was sponsored by Astellas Pharma Korea, Inc. Editorial assistance was funded by Astellas Pharma Korea, Inc.

\section{Additional Contributions}

We would like to thank Hye-Ryon Kim (Medical Writing; www. medicalwriting. co. kr) and Dr Szymon Bruzewicz (SciencePro; www. sciencepro. co. kr) for assistance in drafting and revising the manuscript under the direction of the authors. Editorial support was provided by Cello Health MedErgy.

\section{ORCID}

Jinsoo Rhu 
Kyo Won Lee

https://orcid.org/0000-0002-2722-7817

Young Jae Chung

https://orcid.org/0000-0003-0725-1040

Jae Berm Park

https://orcid.org/0000-0001-9117-2278

Ju Young Choi

https://orcid.org/0000-0002-1121-726X

Sung Joo Kim

https://orcid. org/0000-0002-2584-4483

Jin Sang Jung

Sehyun Kim

https://orcid.org/0000-0002-1931-7169

\section{Author Contributions}

Conceptualization: JR, SJK, JBP, SK. Data curation: SK. Formal analysis: SK. Funding acquisition: JSJ. Investigation: JR, KWL, YJC, JBP, JYC, SJK. Methodology: SK, JR, SJK, JBP, SK. Project administration: JSJ, JYC. Writing - original draft: JR, SJK, JBP, SK, JSJ. Writing - review \& editing: all authors.

\section{Additional Information}

Researchers requested access to anonymized participantlevel data, trial-level data, and protocols from Astellas sponsored clinical trials at www. clinicalstudydatarequest. com. For the Astellas criteria on data sharing, see: https:// clinicalstudydatarequest. com/Study-Sponsors/Study-Spo nsors-Astellas. aspx.

\section{REFERENCES}

1. Coemans M, Süsal C, Döhler B, Anglicheau D, Giral M, Bestard O, et al. Analyses of the short- and long-term graft survival after kidney transplantation in Europe between 1986 and 2015. Kidney Int 2018;94:964-73.

2. Wiebe C, Gibson IW, Blydt-Hansen TD, Karpinski M, Ho J, Storsley LJ, et al. Evolution and clinical pathologic correlations of de novo donor-specific HLA antibody post kidney transplant. Am J Transplant 2012;12:1157-67.

3. Wiebe C, Gibson IW, Blydt-Hansen TD, Pochinco D, Birk PE, Ho J, et al. Rates and determinants of progression to graft failure in kidney allograft recipients with de novo donor-specific antibody. Am J Transplant 2015; 15:2921-30.

4. Kuypers DR, Peeters PC, Sennesael JJ, Kianda MN, Vrijens B, Kristanto $\mathrm{P}$, et al. Improved adherence to tacrolimus once-daily formulation in renal recipients: a randomized controlled trial using electronic monitoring. Transplantation 2013;95:333-40.

5. Testa MA, Simonson DC. Assessment of quality-of-life

outcomes. N Engl J Med 1996;334:835-40.

6. Fiebiger W, Mitterbauer C, Oberbauer R. Health-related quality of life outcomes after kidney transplantation. Health Qual Life Outcomes 2004;2:2.

7. Finkelstein FO, Wuerth D, Finkelstein SH. Health related quality of life and the CKD patient: challenges for the nephrology community. Kidney Int 2009;76:946-52.

8. Chisholm-Burns M, Pinsky B, Parker G, Johnson P, Arcona S, Buzinec $\mathrm{P}$, et al. Factors related to immunosuppressant medication adherence in renal transplant recipients. Clin Transplant 2012;26:706-13.

9. Sabbatini M, Garofalo G, Borrelli S, Vitale S, Torino $\mathrm{M}$, Capone D, et al. Efficacy of a reduced pill burden on therapeutic adherence to calcineurin inhibitors in renal transplant recipients: an observational study. Patient Prefer Adherence 2014;8:73-81.

10. Shabany Hamedan M, Mohamad Aliha J. Relationship between immunosuppressive medications adherence and quality of life and some patient factors in renal transplant patients in Iran. Glob J Health Sci 2014;6:205-12.

11. Scheel JF, Schieber K, Reber S, Stoessel L, Waldmann $\mathrm{E}$, Jank S, et al. Psychosocial variables associated with immunosuppressive medication non-adherence after renal transplantation. Front Psychiatry 2018;9:23.

12. Laupacis A, Pus N, Muirhead N, Wong C, Ferguson B, Keown P. Disease-specific questionnaire for patients with a renal transplant. Nephron 1993;64:226-31.

13. Laupacis A, Muirhead N, Keown P, Wong C. A disease-specific questionnaire for assessing quality of life in patients on hemodialysis. Nephron 1992;60:302-6.

14. Hays RD, Kallich JD, Mapes DL, Coons SJ, Carter WB. Development of the kidney disease quality of life (KDQOL) instrument. Qual Life Res 1994;3:329-38.

15. Franke GH, Reimer J, Kohnle M, Luetkes P, Maehner N, Heemann U. Quality of life in end-stage renal disease patients after successful kidney transplantation: development of the ESRD symptom checklist - transplantation module. Nephron 1999;83:31-9.

16. Guillemin F, Bombardier C, Beaton D. Cross-cultural adaptation of health-related quality of life measures: literature review and proposed guidelines. J Clin Epidemiol 1993;46:1417-32.

17. Beaton DE, Bombardier C, Guillemin F, Ferraz MB. Guidelines for the process of cross-cultural adaptation of self-report measures. Spine (Phila Pa 1976) 2000; 25:3186-91.

18. Reichenheim ME, Moraes CL. Operationalizing the cross-cultural adaptation of epidemiological measurement instruments. Rev Saude Publica 2007;41:665-73. 
19. Braun V, Clarke V. Using thematic analysis in psychology. Qual Res Psychol 2006;3:77-101.

20. Carmines EG, Zeller RA. Reliability and validity assessment. Thousand Oaks, CA: SAGE Publications; 1979.

21. Cronbach LJ. Coefficient alpha and the internal structure of tests. Psychometrika 1951;16:297-334.

22. Streiner DL, Norman GR, Cairney J. Health measurement scales: a practical guide to their development and use. New York, NY: Oxford University Press; 2015.

23. Park HJ, Kim S, Yong JS, Han SS, Yang DH, Meguro $\mathrm{M}$, et al. Reliability and validity of the Korean version of Kidney Disease Quality of Life instrument (KDQOLSF). Tohoku J Exp Med 2007;211:321-9.

24. Hays RD, Kallich J, Mapes D, Coons S, Amin N, Carter WB, et al. Kidney Disease Quality of Life Short Form (KDQOL-SFTM), Version 1.3 [Internet]. Santa Monica, CA: RAND Corporation; 1997 [cited 2019 Dec 20]. Available from: https://www. rand. org/pubs/papers/P7994. html.
25. Rebollo P, Ortega F, Ortega T, Valdés C, GarcíaMendoza M, Gómez E. Spanish validation of the "kidney transplant questionnaire" : a useful instrument for assessing health related quality of life in kidney transplant patients. Health Qual Life Outcomes 2003;1:56.

26. Wang WL, Lee HL, Fetzer SJ. Challenges and strategies of instrument translation. West J Nurs Res 2006;28: 310-21.

27. Rebafka A. Medication adherence after renal transplantation-a review of the literature. J Ren Care 2016;42:239-56.

28. Morisky DE, Ang A, Krousel-Wood M, Ward HJ. Predictive validity of a medication adherence measure in an outpatient setting. J Clin Hypertens (Greenwich) 2008; 10:348-54.

29. Mouelhi Y, Jouve E, Alessandrini M, Pedinielli N, Moal $\mathrm{V}$, Meurette A, et al. Factors associated with healthrelated quality of life in kidney transplant recipients in France. BMC Nephrol 2018;19:99. 